\title{
Preface to the CLEI 2018 Special Issue
}

We are proud to introduce the special issue with extended versions of selected papers presented in the 43rd Latin American Conference in Informatics (CLEI 2018). CLEI received more than 340 submissions, of which circa 100 papers were accepted and presented during the event, held in São Paulo, Brazil. This special issue contains papers selected among those presented in the conference, considering its quality and contribution. Then, the authors were invited to include novel contributions to the papers, and went through another round of review. A total of 8 papers were included in this special issue, from a variety of topics, reflecting the diversity of research lines conveyed in the conference:

1. Feature Selection for Clustering of Homicide Rates in the Brazilian State of Goias

2. Filtered-ARN: Asymmetric objective measures applied to filter Association Rules Networks

3. Classical Machine Learning Techniques in the Search of Extrasolar Planets

4. MoFQA: A TDD Process and Tool for Automatic Test Case Generation from MDD Models

5. A New Statistical and Verbal-Semantic Approach to Pattern Extraction in Text Mining Applications

6. An emotional model for swarm robotics

7. Automatic Challenge Generation for Teaching Computer Security

8. Medical Terminology Server for the Hospital of Clinics of Paraguay using Apache Lucene and the UMLS Metathesaurus

The issue is completed with one regular paper:

9. Multi-objective Evolutionary Algorithms for Power Distribution System Optimal Reconfiguration

We would like to thank all the members of the community which were involved in this issue, and particularly the reviewers for their anonymous contribution to the selection process which ensured the quality of the selection.

Adenilso da Silva Simão, Valeria Farinazzo Martins \& Héctor Cancela

Special Issue Editors 